\title{
Widening the sharpness modulation region of an entanglement-assisted sequential quantum random access code: Theory, experiment, and application
}

\author{
Ya Xiao, ${ }^{*}$ Xin-Hong Han, Xuan Fan, Hui-Chao Qu, and Yong-Jian Gu $\odot^{\dagger}$ \\ Department of Physics, Ocean University of China, Qingdao 266100, People's Republic of China
}

(Received 31 January 2021; accepted 15 April 2021; published 29 April 2021)

\begin{abstract}
The sequential quantum random access code (QRAC) allows two or more decoders to obtain a desired message with higher success probability than the best classical bounds by appropriately modulating the measurement sharpness. Here, we propose an entanglement-assisted sequential QRAC protocol which can enable deviceindependent tasks. By relaxing the equal sharpness and mutually unbiased measurement limits, we widen the sharpness modulation region from a one-dimensional interval to a two-dimensional triangle. Then, we demonstrate our scheme experimentally and get more than 27 standard deviations above the classical bound even when both decoders perform approximately projective measurements. We use the observed success probability to quantify the connection among sequential QRAC, measurement sharpness, measurement biasedness, and measurement incompatibility. Finally, we show that our protocol can be applied to sequential device-independent randomness expansion and our measurement strategy can enhance the success probability of decoding the entire input string. Our results may promote a deeper understanding of the relationship among quantum correlation, quantum measurement, and quantum information processing.
\end{abstract}

DOI: 10.1103/PhysRevResearch.3.023081

\section{INTRODUCTION}

The encoding-decoding process is of vital importance in security communication. How information can be encoded in a physical system and how much information can be retrieved lie at the core of communication theory. The random access code (RAC) is a communication protocol which allows us to encode an $m$-bit-long message into a shorter message of $n$ bits such that any one of the $m$ bits can be recovered with a success probability no less than $1 / 2$. It has been shown that the probability can be increased if qubits are employed in encoding messages instead of classical bits [1]. Such a quantum random access code (QRAC) was first introduced for a simple preparation-and-measurement qubit system [1-4], and later developed for entanglement [5,6] and higher-dimensional quantum systems [7]. The diversity of the QRAC protocol provides a wide range of applications in network coding [8], quantum key distribution [9,10], random number generation [11], preparation contextuality [2], dimension witnessing [12], self-testing [13-15], and so on.

To date, most discussions on decoding operators of the QRAC protocol have been focusing on sharp (projective) measurement. This is because the sharper the measurement, the more information is obtained to decode a desired message. However, projective measurement may completely destroy the initial encoded state, so that at most one decoder can perform

\footnotetext{
*xiaoya@ouc.edu.cn

†yjgu@ouc.edu.cn
}

Published by the American Physical Society under the terms of the Creative Commons Attribution 4.0 International license. Further distribution of this work must maintain attribution to the author(s) and the published article's title, journal citation, and DOI. better than the best classical RAC. Recently, sequential unsharp (weak) measurement has attracted much attention. It has already been shown that by appropriately modulating the measurement sharpness, one can obtain enough information without disturbing the initial quantum correlation severely and others are capable of sharing the correlation simultaneously $[16,17]$. Notably, it has been demonstrated theoretically and experimentally that multiple independent parties can share the nonlocality of a single maximally entangled qubit pair [18-20]. Moreover, unsharp measurements have been applied in quantum state tomography [21], incompatible observables [22], and contextuality [23,24], and generate a long sequential sharing of other types of quantum correlations such as entanglement [25], steering [26], and quantum coherence [27].

These advances make it possible to develop protocols for multiple QRAC inequality violations over sequential measurements, which was confirmed by Mohan, Tavakoli, and Brunner (MTB) [28]. They found that two sequential decoders who can successfully decode a message with a nontrivial probability beat the classical limits in the $2 \rightarrow 1$ QRAC scenario. In MTB's protocol, the encoder Alice encodes her two classical bits into one qubit and sends it to the first decoder, Bob. Then Bob applies quantum measurements unsharply, records the results, and relays the resulting postmeasurement states to the second decoder, Charlie, who performs projective measurements on the received qubits. For Bob to successfully decode a message with a nontrivial probability beyond classical limits, the measurements must remain sharp; on the other hand, it should also be unsharp enough to retain some information for Charlie's measurement to make another QRAC inequality violation possible. In 2019, Mohan et al. derived an optimal trade-off relation between these two QRACs, finding that both QRACs can outperform the 
optimal classical bound when Bob's measurement sharpness $\eta \in(1 / \sqrt{2},(2+\sqrt{2}) / 4)$, which has been demonstrated experimentally $[15,29]$.

It should be noted that there are some drawbacks in MTB's protocol which narrow the double QRAC inequality violation region. First, in the choice of Bob's measurement direction, only the maximization of the probability between Alice and Bob $\left(P_{A B}\right)$ is considered, which will unavoidably lower the upper bound of the probability between Alice and Charlie $\left(P_{A C}\right)$. In fact, both QRAC probabilities should be considered to achieve a double QRAC inequality violation. Second, there is an implicit assumption that the sharpness of Bob's two measurements is equal. However, the adoption of unequal sharpness measurements may be more favorable, for instance, by increasing the sequential length; i.e., more Bobs are allowed to share quantum correlation with Alice [19].

In this work, we first extend MTB's protocol into an entanglement-assisted scheme since entanglement-assisted QRAC performs better than a single-photon QRAC [5,6,30] and has application for full device-independent quantum information tasks [31]. Then, we introduce a method to widen the useful region of the measurement sharpness parameter from a one-dimensional interval to a two-dimensional triangle to achieve double QRAC inequality violation. Experimentally, we verify the robustness and efficiency of our scheme and further illustrate how it works by quantifying the relationship among the degree of measurement sharpness, biasedness, and incompatibility with the observed two QRAC success probabilities. Finally, we present two specific applications of our protocol: sequential device-independent randomness expansion and increase in the probability of successful decoding of the whole encoded string.

\section{THEORETICAL MODEL}

We start with a brief review of MTB's QRAC protocol. In their protocol, Alice encodes her two classical bits into one of four pure states, which form the vertices of a square in the equatorial line of the Bloch sphere, and then sequentially transmits it to Bob and Charlie to measure [28]. Here, we extend it into an entanglement-assisted scheme where the encoding messages are hidden in the entanglement state shared between Alice and Bob (Charlie) rather than in a proper qubit. The process of communication between Alice and Bob (Charlie) is completed by measuring each qubit on its own part [6].

To clarify our entanglement-assisted sequential QRAC scheme, we show the encoding-decoding process in the case of $2 \rightarrow 1$ in Fig. 1(a). Two decoders, Bob and Charlie, attempt to recover a desired bit encoded in Alice's string with a nontrivial success probability using only a pair of entangled particles. We denote the measurement direction of Alice, Bob, and Charlie by the Bloch vectors $\vec{r}_{x}, \vec{s}_{y}$, and $\vec{t}_{z}$, respectively. To begin with, a pair of two-qubit entanglement particles $\rho_{A B}$ are shared between Alice and Bob. Alice encodes her two classical bits, $x_{0}, x_{1} \in\{0,1\}$, by performing a local measurement $M_{a \mid \vec{r}_{x}}$ on her particle based on the value of $x=x_{0} \oplus x_{1}$. The corresponding measurement result is denoted as $a \in\{0,1\}$. After the measurement, she separately sends one classical bit $m_{1}=x_{0} \oplus a$ to Bob and Charlie, which will help them obtain a higher success decoding probability. In the decoding
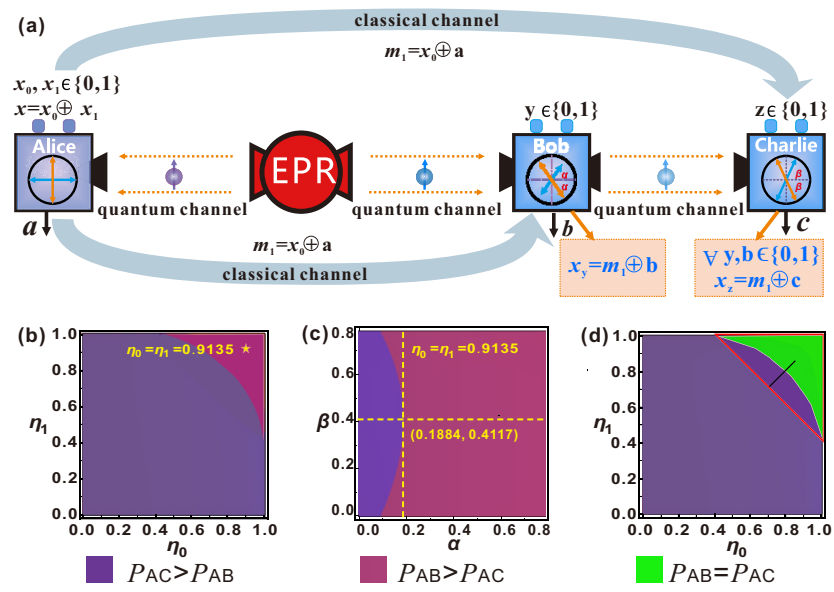

FIG. 1. (a) Entanglement-assisted quantum random access code via sequential measurement. The inserted circles represent our measurement settings; Bob and Charlie implement a separate QRAC with Alice. (b) The relationship between the average success probabilities $\left\{P_{A B}, P_{A C}\right\}$ and Bob's two sharpness parameters $\left\{\eta_{0}, \eta_{1}\right\}$ when the measurement directions of Bob and Charlie both are set at $\alpha=\beta=$ $\pi / 4$. (c) The optimal choice of measurements for Bob and Charlie when $\eta_{0}=\eta_{1}=0.9135$ [marked as a star in (b)]. (d) The relationship between the average success probabilities $\left\{P_{A B}, P_{A C}\right\}$ and Bob's two sharpness parameters $\left\{\eta_{0}, \eta_{1},\right\}$ when Bob and Charlie further optimize their measurement directions by maximizing the minimum value of $\left\{P_{A B}, P_{A C}\right\}$. The area enclosed by the red curve corresponds to the double QRAC inequality violation area, i.e., $P_{A B} \geqslant 3 / 4$ and $P_{A C} \geqslant 3 / 4$, in our measurement strategy. The black line represents the range of MTB's measurement strategy. See Fig. 3(a) for more details.

process, Bob chooses an unsharp measurement $M_{b \mid \vec{s}_{y}}=$ $K_{b \mid \vec{s}_{y}}^{\dagger} K_{b \mid \vec{s}_{y}}$ according to an input $y \in\{0,1\}$ to decode a desired bit $x_{y} . K_{b \mid \vec{s}_{y}}$ is the corresponding Kraus operator. Then, he records the output result $b \in\{0,1\}$ and compares it with the received bit $m_{1}$. The decoding process is successful if $x_{y}=m_{1} \oplus b$, and the success probability can be calculated by $P\left(x_{y}=m_{1} \oplus b \mid x, y\right)=\operatorname{Tr}\left[M_{a \mid \vec{r}_{x}} \otimes M_{b \mid \vec{s}_{y}} \cdot \rho_{A B}\right]$. Similarly, Charlie receives an input $z \in\{0,1\}$ with an aim to guess $x_{z}$ by taking an optimal measurement $M_{c \mid \vec{t}_{z}}$ on the particle he received from Bob, yielding an outcome $c \in\{0,1\}$. Since Bob and Charlie separately implement a QRAC with Alice, they act independently. It means Charlie is unaware about what measurements are performed by Bob; thus we have to take the average of Bob's input and output to obtain the state shared between Alice and Charlie, which is given by $\rho_{A C}=$ $\frac{1}{2} \sum_{y, b}\left(I_{A} \otimes K_{b \mid \vec{s}_{y}}\right) \rho_{A B}\left(I_{A} \otimes K_{b \mid \vec{s}_{y}}^{\dagger}\right)$. Charlie's success probability then can be given as $P\left(x_{z}=m_{1} \oplus c \mid x, z\right)=\operatorname{Tr}\left[M_{a \mid \vec{r}_{x}} \otimes\right.$ $\left.M_{c \mid \vec{t}_{z}} \cdot \rho_{A C}\right]$. Suppose all the inputs $\left(x_{0}, x_{1}, y, z\right)$ are statistically independent and uniformly distributed. Repeating the process many times, the average success probability of Bob and Charlie, $P_{A B}$ and $P_{A C}$, can be obtained by

$$
\begin{aligned}
& P_{A B}=\frac{1}{8} \sum_{x, y} P\left(x_{y}=m_{1} \oplus b \mid x, y\right), \\
& P_{A C}=\frac{1}{8} \sum_{x, z} P\left(x_{z}=m_{1} \oplus c \mid x, z\right) .
\end{aligned}
$$


The optimal classical limit of both average success probabilities is $3 / 4$ [1]. When Alice and Bob (Charlie) behave classically, then $P_{A B} \leqslant 3 / 4 \quad\left(P_{A C} \leqslant 3 / 4\right)$, which we call QRAC inequality. It has been demonstrated that the double QRAC inequality violation can be observed simultaneously by adjusting Bob's measurement sharpness parameter and its useful range is $(1 / \sqrt{2},(2+\sqrt{2}) / 4)$ [28]. In the following, we will show that the range can be expanded by optimizing the measurement settings of both decoders.

As the MTB QRAC protocol, we assume Alice encodes her two classical bits in the $X O Z$ plane. For convenience, we suppose Alice performs measurement along $X$ and $Z$ axes. It is obvious that the optimal decoding operators need to be performed on the same plane [13]. Thus, the decoding measurement direction for Bob and Charlie can be expressed as $\cos \alpha X \pm \sin \alpha Z$ and $\cos \beta X \pm \sin \beta Z$, respectively [as shown in Fig. 1(a)]. Here, we relax the equal sharpness and mutually unbiased conditions of each decoder's respective pair of measurements in the previous schemes [15,28,29]. Then the measurement settings of each encoder and decoder can be rewritten as

$$
\begin{aligned}
M_{a \mid \vec{r}_{x}} & =\left[I_{A}+(-1)^{a}\left((x \oplus 1) \sigma_{1}+x \sigma_{3}\right)\right] / 2, \\
M_{b \mid \vec{s}_{y}} & =\left[I_{B}+(-1)^{b} \eta_{y}\left(\cos \alpha \sigma_{1}+(-1)^{y} \sin \alpha \sigma_{3}\right)\right] / 2, \\
M_{c \mid \vec{t}_{z}} & =\left[I_{C}+(-1)^{c}\left(\cos \beta \sigma_{1}+(-1)^{z} \sin \beta \sigma_{3}\right)\right] / 2,
\end{aligned}
$$

where $\eta_{y} \in(0,1)$ is the sharpness parameter. With these measurement settings and an initial maximum entanglement state, the expected average success probabilities of Bob and Charlie, $P_{A B}$ and $P_{A C}$, can be expressed as

$$
\begin{aligned}
& P_{A B}=\frac{1}{8}\left[4+\left(\eta_{0}+\eta_{1}\right)(\cos \alpha+\sin \alpha)\right], \\
& P_{A C}=\frac{1}{8}\left[4+2 M+N\left(\sqrt{1-\eta_{0}^{2}}+\sqrt{1-\eta_{1}^{2}}\right)\right],
\end{aligned}
$$

where $M=\cos ^{2} \alpha \cos \beta+\sin ^{2} \alpha \sin \beta, N=\cos ^{2} \alpha \sin \beta+$ $\sin ^{2} \alpha \cos \beta, \alpha, \beta \in[0, \pi / 4]$. Specifically, when $\eta_{0}=\eta_{1}=\eta$ and $\alpha=\beta=\pi / 4$, our scheme goes back to an entanglementassisted MTB protocol with the same sharpness modulation range, $\eta \in(1 / \sqrt{2},(2+\sqrt{2}) / 4)$.

It easy to find that, from Eq. (3), for a given pair of values of $\eta_{0}$ and $\eta_{1}$, once $\alpha$ is set, not only can $P_{A B}$ be obtained, but also a proper value of $\beta$ can be found to maximize the value of $P_{A C}$. In order to keep both probabilities surpassing the optimal classical limit within a wider sharpness region, the best way is to choose a pair of $\{\alpha, \beta\}$ that maximizes the minimum value of $\left\{P_{A B}, P_{A C}\right\}$. Noting that the maximum value of $P_{A B}$ is always obtained at $\alpha=\pi / 4$ once $\eta_{0}$ and $\eta_{1}$ are set, and if $\beta$ is updated to maximize $P_{A C}, P_{A C}$ will increase with a gradual decrease of $\alpha$ from $\pi / 4$ to zero, and $P_{A B}$ will decrease at the same time. Thus, for each pair of $\left\{\eta_{0}, \eta_{1}\right\}$, we start with $\alpha=\pi / 4$, then maximize the value of $P_{A C}$, and finally compare the values of $P_{A B}$ and $P_{A C}$. If $P_{A B} \leqslant P_{A C}$, the current values of $\{\alpha, \beta\}$ are set as the optimal measurement parameters for Bob and Charlie. This is because, in this case, maximizing the minimum value of $\left\{P_{A B}, P_{A C}\right\}$ is equal to maximizing the value of $P_{A B}$ which can be realized by setting $\alpha=\pi / 4$, and in the meantime the maximum value of $P_{A C}$ can be obtained with $\beta=\pi / 4$. Clearly, when $P_{A B} \leqslant P_{A C}$, both Bob's and Charlie's measurement directions are same as

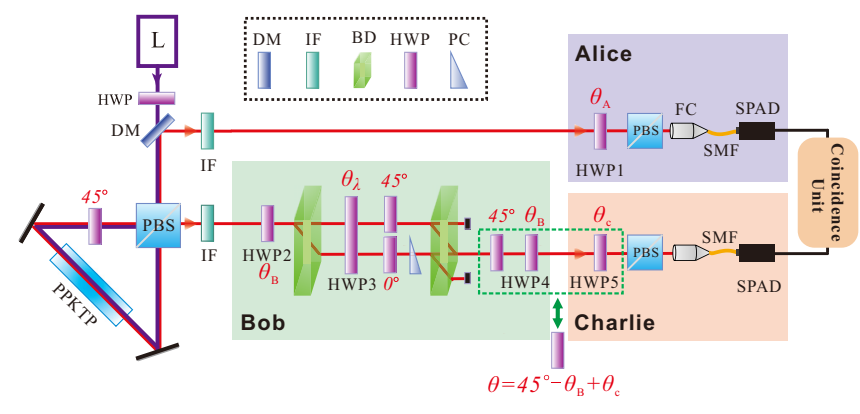

FIG. 2. Experimental setup. A bright source of polarizationentangled photon pairs in a state $(|H H\rangle+|V V\rangle) / \sqrt{2}$ is generated via the spontaneous parametric down-conversion process by pumping a type-II cut PPKTP crystal located in a Sagnac interferometer with an ultraviolet laser (L) at $405 \mathrm{~nm}$. These two photons are filtered by interference filters (IFs). One of the photons is sent to Alice, and the other one is subsequently sent to Bob and Charlie. A balanced Mach-Zehnder interferometer (MZI) consisting of two calcite beam displacers (BDs) and a couple of half-wave plates (HWPs) is used to realize Bob's unsharp measurement, where the sharpness parameter $\eta_{y}=\cos \left(4 \theta_{\lambda}\right)$ can be tuned conveniently by rotating the internal HWP3. HWP2 and HWP4 are used to change any of $\left\{M_{0 \mid \vec{s}_{0}}, M_{1 \mid \vec{s}_{0}}, M_{0 \mid \vec{s}_{1}}, M_{1 \mid \vec{s}_{1}}\right\}$ into another as well as selecting the basis and outcome of the measurement. The relative phase between the existing two paths can be controlled conveniently by employing a removable wedge phase plate (PC). Both Alice and Charlie only perform projective measurements in the space of linear polarization; hence their setup can be simplified to composition of a HWP and a polarization beam splitter (PBS). To reduce the number of components, we replace the group of three consecutive HWPs in the green dotted box with a single HWP. The photons are detected by singlephoton detectors (SPADs) and the signals are sent for coincidence.

MTB's protocol; otherwise, if $P_{A B}>P_{A C}$ when $\alpha=\pi / 4$, we have to optimize their measurement directions to increase the value of $P_{A C}$. Here, we gradually decrease the value of $\alpha$, then choose a proper value of $\beta$ to maximize $P_{A C}$ for each given $\alpha$. The maximum value of the minimum value of $\left\{P_{A B}, P_{A C}\right\}$ can be obtained when $P_{A B}=P_{A C}$. Figure 1(c) shows the optimal value of $\alpha$ and $\beta$ in the case of $\eta_{0}=\eta_{1}=0.9135$. Similarly, for other given pairs of $\left\{\eta_{0}, \eta_{1}\right\}$, we can always find the optimal numerical solutions of $\{\alpha, \beta\}$ (see Appendix A for more details). Eventually, we can further extend the double QRAC inequality violation region as the area enclosed by the red line in Fig. 1(d), which is much larger than the original range $\eta_{0}=\eta_{1} \in(1 / \sqrt{2},(2+\sqrt{2}) / 4)$, the black line.

\section{EXPERIMENTAL METHOD}

To verify the advantage of our protocol, we put it into practice with an optic experiment. Figure 2 shows our experimental setup. A 405-nm continuous-wave diode laser (L) is used to pump a 15-mm-long periodically poled KTP (PPKTP) crystal inside a polarization Sagnac interferometer in clockwise and counterclockwise directions to generate a maximum two-qubit polarization-entangled photons state $(|H H\rangle+|V V\rangle) / \sqrt{2}$ [32]. Two interference filters (IFs) with bandwidth of $3 \mathrm{~nm}$ are used to filter these photons. One of the two photons is sent to Alice to 
encode her two classical bits, and the other photon is sequentially sent to two decoders, Bob and Charlie. During the encoding-decoding process, Alice encodes her two classical bits into her measurement by using a combination of halfwave plate (HWP1) and polarized beam splitter (PBS), shown in the light blue green region. The angle of HWP1 is set at $22.5^{\circ},-22.5^{\circ}, 0^{\circ}$, and $45^{\circ}$ for $M_{0 \mid \vec{r}_{0}}, M_{1 \mid \vec{r}_{0}}, M_{0 \mid \vec{r}_{1}}$, and $M_{1 \mid \vec{r}_{1}}$, respectively. Based on Alice's measurement, Bob and Charlie select an optimal measurement acting on their own photon to decode a desired bit.

Bob's unsharp measurement can be realized by using a balanced Mach-Zehnder interferometer (MZI), equipped with some HWPs and two calcite beam displacers (BDs) which transmit vertical polarization $|V\rangle$ and displace horizontal polarization $|H\rangle$ with $3 \mathrm{~mm}$ (light green region) $[15,17,18,26,33]$. In each round of the experiment, HWP2 is set at a special angle $\theta_{B}$ to transform the eigenbases, $\left\{|\varphi\rangle,\left|\varphi^{\perp}\right\rangle\right\}$, of Bob's current operator to the $\{H, V\}$ basis. Here $|\varphi\rangle\left(\left|\varphi^{\perp}\right\rangle\right)$ represents the eigenstate with an outcome equal to zero (1). More clearly, $|\varphi\rangle \rightarrow|H\rangle$ and $\left|\varphi^{\perp}\right\rangle \rightarrow|V\rangle$. Then an unsharp measurement of observable $\Pi=|H\rangle\langle H|-$ $|V\rangle\langle V|$ was performed with the elements between HWP2 and HWP4, and finally transformed back to Bob's measurement basis via HWP4 by rotating the same angle as HWP2. A HWP3 spanning across both arms is used to tune the measurement sharpness parameter through changing its angle $\theta_{\lambda}$. For simplicity of the experimental implementation, we only extract the measurement outcome of $b=0$ by postselecting a particular output mode of the interferometer (the blocked mode corresponding to $b=1)$. Thus after the setup, a Kraus operator $K_{0 \mid \vec{s}_{\nu}}=\cos \left(2 \theta_{\lambda}\right)|\varphi\rangle\left\langle\varphi\left|+\sin \left(2 \theta_{\lambda}\right)\right| \varphi^{\perp}\right\rangle\left\langle\varphi^{\perp}\right|$ corresponding to the current unsharp measurement $M_{0 \mid \vec{s}_{y}}$ is realized, where $M_{0 \mid \vec{s}_{y}}=K_{0 \mid \vec{s}_{y}}^{\dagger} K_{0 \mid \vec{s}_{y}}$ and $\eta_{y}=\cos \left(4 \theta_{\lambda}\right)$. Likewise, one just needs to replace the rotating operation associated with the wave plates (HWP2, HWP4) by $|\varphi\rangle \rightarrow|V\rangle$ and $\left|\varphi^{\perp}\right\rangle \rightarrow$ $|H\rangle$; then the other Kraus operator $K_{1 \mid \vec{s}_{y}}=\sin \left(2 \theta_{\lambda}\right)|\varphi\rangle\langle\varphi|+$ $\cos \left(2 \theta_{\lambda}\right)\left|\varphi^{\perp}\right\rangle\left\langle\varphi^{\perp}\right|$ of the other required unsharp measurement $M_{1 \mid \vec{s}_{y}}=K_{1 \mid \vec{s}_{y}}^{\dagger} K_{1 \mid \vec{s}_{y}}$ can also be obtained. Thus, depending on the orientation of these plates, the interferometer can carry out each of the unsharp operators $\left\{M_{0 \mid \vec{s}_{0}}, M_{1 \mid \vec{s}_{0}}, M_{0 \mid \vec{s}_{1}}, M_{1 \mid \vec{s}_{1}}\right\}$ required by the protocol.

Charlie's measurements are projective and, therefore, his setup only consists of a HWP preceded by a PBS (light red region). To reduce the number of components, we replaced the group of three consecutive HWPs with a single HWP (dotted green box). The equivalent relationship of the angles of these wave plates is shown in Fig. 2. A single-mode fiber finally collects Charlie's photons and brings them to a SPAD, the signal of which is correlated with Alice's signal by a coincidence unit.

In our experiment, we choose 12 different values for Bob's two measurement sharpness parameters $\eta_{0}$ and $\eta_{1}$, separately. Both of them are almost equidistantly distributed in $[0,1]$. Based on the measurement strategy introduced in the previous section, HWP2, HWP4, and HWP5 choose an appropriate angle from $\left\{-33.75^{\circ},-11.25^{\circ}, 11.25^{\circ}, 33.75^{\circ}\right\}$ based on the initial bit that Bob and Charlie desire to decode when $P_{A B} \leqslant$ $P_{A C}$. Otherwise, we should optimize these angles to maximize the minimum value of $\left\{P_{A B}, P_{A C}\right\}$ for a given $\left\{\eta_{0}, \eta_{1}\right\}$. Special settings of wave plates in the case of $\eta_{0}=0.707$ and $\eta_{0}=$ $\eta_{1}$ are listed in Appendix A. During the encoding-decoding process, each of Alice, Bob, and Charlie has two measurement choices, and for each choice, two trials are needed, corresponding to two different outcomes. Thus, for each fixed $\left\{\eta_{0}, \eta_{1}\right\}$, we have implemented 32 trials for calculating $P_{A B}$ and 64 trials for calculating $P_{A C}$. And for each trial, we record the coincidence counting events for $4 \mathrm{~s}$. The total number of coincident events contributing to a complete measurement is about $4 \times 10^{5}$, sufficient to make the statistical errors small, which is about $1 \times 10^{-3}$ in our experiment.

\section{EXPERIMENTAL RESULTS}

The experimental average success probabilities $P_{A B}$ (red marks) and $P_{A C}$ (blue marks) for several values of $\eta_{0}$ and $\eta_{1}$ are shown in Fig. 3(a). In general, our experimental results are in good agreement with the theoretical predictions. It is clearly shown that a double outperformed QRAC can be obtained when $\left\{\eta_{0}, \eta_{1}\right\}$ lies on the right side of the intersection line of the light red plane and the light blue plane (green dashed line). And the useful range for one sharpness parameter increases with the increasing of the other sharpness parameter. The measured and theoretical success probabilities are displayed for fixed values of $\eta_{0}=0.707$ in Fig. 3(b) and of $\eta_{0}=\eta_{1}$ in Fig. 3(c). The double QRAC inequality violation range is denoted as the light red area, which is enlarged by the inserted box. In particular, even when Bob performs approximately projective measurements, $\eta_{0}=\eta_{1}=0.99$, both Bob and Charlie can beat the classical limit with $P_{A B}=0.77153 \pm$ 0.00078 and $P_{A C}=0.77093 \pm 0.00071$, which is impossible in the original protocol $[15,28,29]$.

In order to illustrate how our measurement strategy works, we further quantify the degree of measurement sharpness, biasedness, and incompatibility with the observed two QRAC success probabilities, which can be expressed as

$$
\begin{gathered}
\eta \geqslant \eta_{\text {low }}=\sqrt{2}\left(2 P_{A B}-1\right), \\
\eta \leqslant \eta_{\text {up }}=2 \sqrt{\left(2+\sqrt{2}-4 P_{A C}\right)\left(2 P_{A C}-1\right)}, \\
\left|\vec{s}_{0} \cdot \vec{s}_{1}\right| \leqslant s_{\text {up }}=\frac{8 P_{A B}-4}{\eta_{1}+\eta_{2}} \sqrt{2-\left(\frac{8 P_{A B}-4}{\eta_{1}+\eta_{2}}\right)^{2}}, \\
D\left(\vec{s}_{0} \cdot \vec{s}_{1}\right) \geqslant 8 P_{A B}-6, \quad D\left(\vec{t}_{0} \cdot \vec{t}_{1}\right) \geqslant \frac{16 P_{A C}-8}{m}-2,
\end{gathered}
$$

where $m$ represents the maximum distance between Charlie's corresponding conditional states. And we can obtain the upper bound of Charlie's measurement biasedness $\left|\vec{t}_{0} \cdot \vec{t}_{1}\right|$ by substituting $\alpha=\arccos \left(s_{\text {up }}\right) / 2$ into Eq. (3). Theoretically, $\mid \vec{s}_{0} \cdot \vec{s}_{1}=\cos (2 \alpha)$ and $\left|\vec{t}_{0} \cdot \vec{t}_{1}\right|=\cos (2 \beta)$. The detailed theoretical calculation is shown in Appendix B. Figure 3(d) plots the lower and upper bounds of Bob's sharpness parameter as a function of targeted sharpness $\eta_{t}$. The tightness of the bounds prove that our setup realized a perfect positive operator-valued measurement. With the determined sharpness bounds, we can deduce the upper bounds of biasedness parameters, $\left|\vec{s}_{0} \cdot \vec{s}_{1}\right|$ and $\left|\vec{t}_{0} \cdot \vec{t}_{1}\right|$, in Bob's and Charlie's respective pair of measurements, which are also shown in Fig. 3(d). As expected, the measurement biasedness parameters become higher than zero 

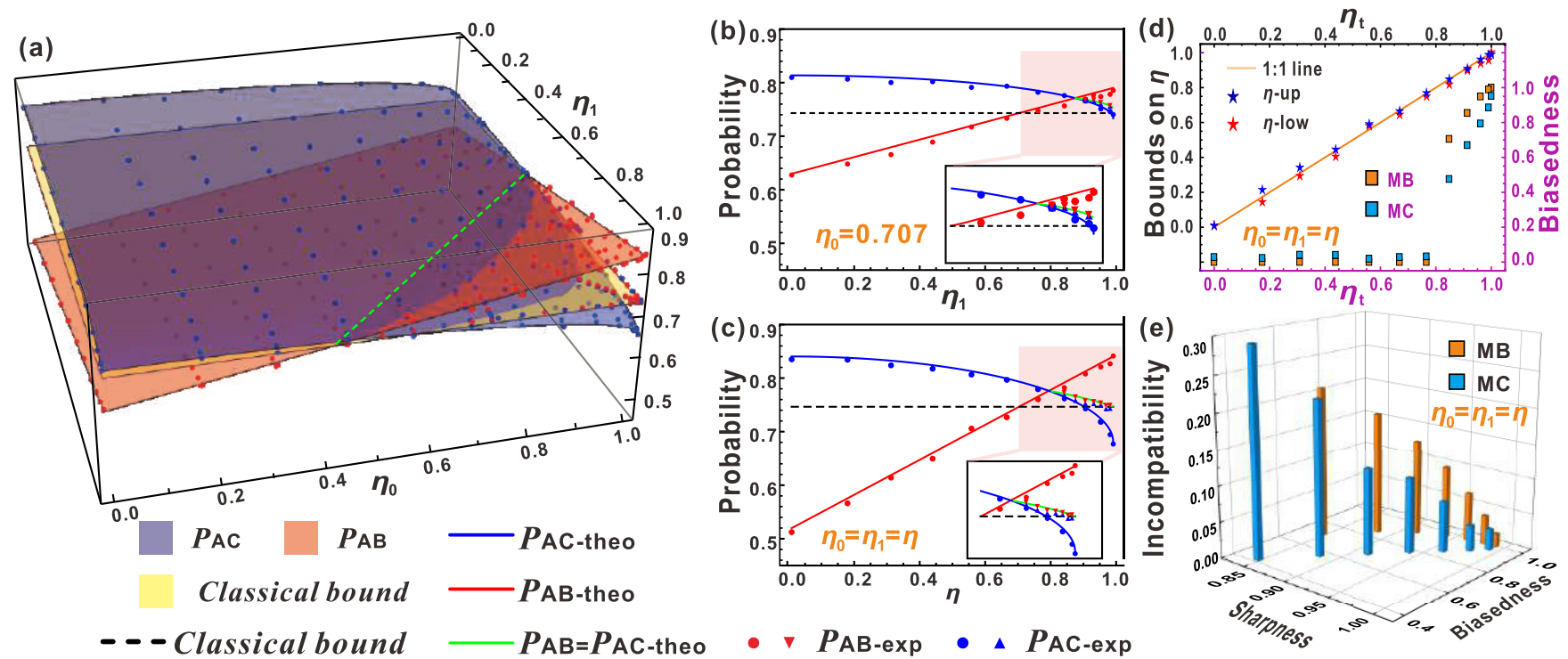

FIG. 3. Demonstrating the double violation of QRAC inequality. (a) The average success probabilities are experimentally measured for various pairs of measurement sharpness parameters $\left\{\eta_{0}, \eta_{1}\right\}$. Experimentally measured average success probabilities are shown as markers, where the red and blue circles (triangles) correspond to $P_{A B}$ and $P_{A C}$ when Bob and Charlie have (have not) further optimizing their measurement directions at $P_{A B}>P_{A C}$. Theoretical predictions are also represented as surface plots, and compared with the experimental data. The average success probabilities are plotted as a function of $\eta_{1}$ for fixed (b) $\eta_{0}=0.707$ and (c) $\eta_{0}=\eta_{1}=\eta$. The shaded areas with light red color indicate the double violation of QRAC inequality. It is clearly demonstrated that the double violation area can be further expanded with the mutually biased measurements. (d) The lower and upper bounds of Bob's sharpness parameter (stars) together with the upper bound of both decoders' measurement biasedness (squares) obtained by the experimental average success probabilities vs the target measurement sharpness. It is obvious that with the increasing of the measurement sharpness, both decoders' biasedness parameter increases but their difference decreases. (e) The relationship between the lower bound on the degree of incompatibility in Bob's (dark yellow) and Charlie's (dark cyan) mutually biased measurements corresponding to seven different values of sharpness and biasedness parameters shown in (c). They are both above zero in the optimizing measurement range and decrease as the sharpness and biasedness parameter increase. Error bars are estimated by the Poissonian statistics of two-photon coincidences which are too small to be visible (see Appendix C for more details).

near $\eta=0.707$, demonstrating that Bob and Charlie need to optimize their measurement direction when $P_{A B}>P_{A C}$. We also quantify the lower bound degree of incompatibility obtained from the seven experimentally measured probability pairs represented as red down-pointing triangles and blue up-pointing triangles in Fig 3(c). Figure 3(e) presents its relationship with the measurement sharpness and biasedness. Obviously, all of the degrees of incompatibility in Bob's and Charlie's respective pair of measurements are above zero, verifying measurement incompatibility is necessary to observe outperforming QRAC [34-36].

\section{APPLICATIONS}

In 2010, Pironio et al. proposed a random number expansion protocol, where the generated randomness can be certified by violating the Clauser-Horne-Shimony-Holt (CHSH) inequality and quantified by min-entropy [31], which reads as

$$
\begin{aligned}
I_{A B} & =\frac{1}{n} \sum_{x, y}(-1)^{x y}[N(a=b \mid x y)-N(a \neq b \mid x y)], \\
& =\left(\eta_{0}+\eta_{1}\right)(\cos \alpha+\cos \beta), \\
H_{\min _{A B}} & =1-\log _{2}\left(1+\sqrt{1}+\frac{I_{A B}^{2}}{4}\right),
\end{aligned}
$$

$$
\begin{aligned}
I_{A C} & =\frac{1}{n} \sum_{x, z}(-1)^{x z}[N(a=c \mid x y)-N(a \neq c \mid x z)], \\
& =2 M+N\left(\sqrt{1-\eta_{0}^{2}}+\sqrt{1-\eta_{1}^{2}}\right), \\
H_{\min _{A C}} & =1-\log _{2}\left(1+\sqrt{1+\frac{I_{A C}^{2}}{4}}\right),
\end{aligned}
$$

where $M$ and $N$ have been defined in Eq. (3), $N(a=b \mid x y)$ is the number of times that the measurements $\{x, y\}$ are performed with outcomes $a$ and $b$ found equal after $n$ runs of the experiment, and $N(a \neq b \mid x y), N(a=c \mid x z)$, and $N(a \neq c \mid x z)$ are defined analogously. Figure 4(a) confirms the validity of Eq. (5) in the cases of $\eta_{0}=\eta_{1}=\eta$ and $\alpha=\beta=\pi / 4$. The observed double $\mathrm{CHSH}$ inequality violation implies that the positive amount of randomness can be generated not only between Alice and Bob but also between Alice and Charlie at the same time. The total min-entropy bound based on entanglement-assisted sequential QRAC as the function of the measurement sharpness is plotted in Fig. 4(a). The result shows that we can always get the positive amount of randomness as soon as $\eta>0$. And the amount of randomness reaches the minimum value during the double $\mathrm{CHSH}$ inequality violation range and achieves the maximum number at the full no-signaling condition, i.e., $\eta \rightarrow 0$ or $\eta \rightarrow 1$. It means the no-signaling condition is critical for guaranteeing the 

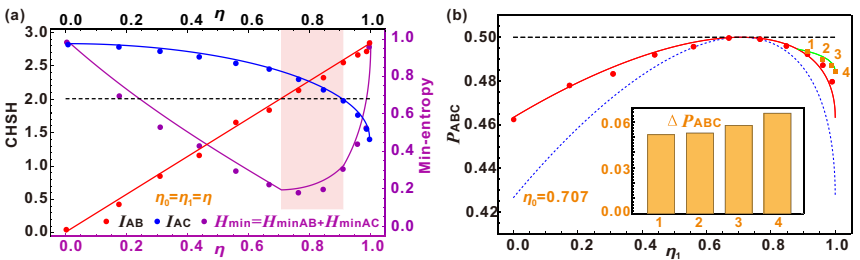

FIG. 4. (a) Device-independent quantum random number expansion by entanglement-assisted sequential QRAC. The light red region represents the double CHSH inequality violation. (b) The decoding probability of Alice's entire input sequence as a function of $\eta_{1}$. The red (green) curve indicates the theoretical probability that Bob and Charlie have (have not) further optimized their measurement directions when $P_{A B}>P_{A C}$. The inset is the increment of decoded probability when Bob and Charlie further optimize their measurement direction. The blue dashed curve represents the probability of MTB's measurement strategy. Error bars are estimated by the Poissonian statistics of two-photon coincidences which are too small to be visible.

randomness; conversely, the decreasing of the total minentropy may imply increasing signaling between Bob and Charlie.

The other application is to increase the decoding probability of Alice's entire input string. When Bob and Charlie cooperate in a communication scenario and agree to always decode different bits, the joint probability of both being correct is given by

$$
\begin{aligned}
P_{A B C}= & \frac{1}{8} \sum_{x, y} P\left(x_{y}=m_{1} \oplus b, x_{z}=m_{1} \oplus c \mid x, y, z \neq y\right), \\
= & \frac{1}{4}+\frac{\cos (\alpha+\beta)}{8}(\cos \alpha-\sin \alpha) \\
& +\frac{\eta_{0}+\eta_{1}}{16}(\cos \alpha+\sin \alpha) \\
& +\frac{1}{16}\left(\sqrt{1-\eta_{0}^{2}}+\sqrt{\left.1-\eta_{1}^{2}\right)}(\cos \alpha+\sin \alpha) \sin (\alpha+\beta) .\right.
\end{aligned}
$$

Figure 4(b) shows the relationship of $P_{A B C}$ versus $\eta_{1}$ when $\eta_{0}=0.707$. It is obvious that our measurement strategy (red and green lines) does allow them to obtain a higher success probability than MTB's strategy (blue dashed line). And the increment can be further improved by optimizing the decoding direction when $P_{A B}>P_{A C}$ [see the inset in Fig. 4(b)].

\section{CONCLUSIONS AND DISCUSSION}

In this work, we theoretically develop and experimentally demonstrate that it is feasible to sustain a double QRAC inequality violation in a much larger sharpness tunable region using only a pair of two-qubit entanglement states by relaxing the equal sharpness and mutually unbiased measurement limits. A larger useful region means stronger noise robustness, which makes it easier for our measurement strategy to realize quantum correlation sharing in practice. Specifically, we observe a counterintuitive result that the double QRAC inequality violation can be achieved even when both encoders take approximately projective measurements. With the ob- served success probabilities, we further present a quantitative analysis of the principle of our measurement strategy. Our protocol is based on entangled photon pairs, which is important for tasks that require certified entanglement. Here, we provide a potential application for full device-independent sequential random number expansion. Additionally, we experimentally demonstrate that our optimal measurement strategy can further increase the success probability for Bob and Charlie to cooperate in decoding the entirety of Alice's string. Our results provide a more essential way to understand the connection between quantum correlation, quantum measurement, and quantum information tasks.

It would be interesting to extend our protocol to more general sequential QRAC scenarios involving either more measurement settings or higher dimensions. Another extension is further allowing the encoder to optimize their measurement settings or further permitting the decoders to share more classical information, thus opening up the possibility for future decoders to use adaptive strategies, which may increase the sequence length of outperformed decoders. We will carry out some experiments in these directions in the near future.

\section{ACKNOWLEDGMENTS}

This work was supported by the National Natural Science Foundation Regional Innovation and Development Joint Fund (Grant No. 932021070), the National Natural Science Foundation of China (Grant No. 912122020), the China Postdoctoral Science Foundation (Grant No. 861905020051), the Fundamental Research Funds for the Central Universities (Grants No. 841912027, No. 842041012, and No. 201961009), the Applied Research Project of Postdoctoral Fellows in Qingdao (Grant No. 861905040045), and the Young Talents Project at Ocean University of China (Grant No. 861901013107). The authors thank Xue Bin An and Hong Wei Li for fruitful discussions.

\section{APPENDIX A: MEASUREMENT SETTINGS OF BOB AND CHARLIE IN DIFFERENT SITUATIONS}

In this work, we propose a measurement strategy to expand the useful sharpness range of Bob's measurement to enable both entanglement-assisted sequential QRAC protocols to go over the classical bound by optimizing Bob's and Charlie's measurement setting parameters $\{\alpha, \beta\}$ based on maximizing the minimum value of their average success probabilities $\left\{P_{A B}\right.$, $\left.P_{A C}\right\}$.

As mentioned in the main text, for a fixed pair of sharpness parameters $\left\{\eta_{0}, \eta_{1}\right\}$, if $P_{A B} \leqslant P_{A C}$, we need to maximize $P_{A B}$ which can be achieved by setting $\alpha=\pi / 4$ and, meanwhile, the maximum value of $P_{A C}$ can be obtained at $\beta=\pi / 4$. However, if $P_{A B}>P_{A C}$, we have to adjust their measurement settings to increase the value of $P_{A C}$. We start with $\alpha=\pi / 4$ and then decrease its value gradually; the value of $P_{A B}$ will decrease, and the value of $P_{A C}$ will increase in the meantime if $\beta$ is updated to maximize $P_{A C}$. Thus, with the decreasing of $\alpha$, the minimum value of $\left\{P_{A B}, P_{A C}\right\}$ will continuously increase until $P_{A B}=P_{A C}$. Thus the current values of $\alpha$ and $\beta$ which satisfy $P_{A B}=P_{A C}$ make up the optimal measurement setting 
TABLE I. The measurement settings when $\eta_{0}=1.0$.

\begin{tabular}{cccccccc}
\hline \hline$\theta_{\lambda}$ & $\eta_{1}$ & $\alpha$ & $\beta$ & $\theta_{\lambda}$ & $\eta_{1}$ & $\alpha$ & $\beta$ \\
\hline $0^{\circ}$ & 1.000 & $0.00^{\circ}$ & $0.00^{\circ}$ & $8^{\circ}$ & 0.848 & $6.82^{\circ}$ & $12.55^{\circ}$ \\
$1^{\circ}$ & 0.998 & $0.15^{\circ}$ & $2.00^{\circ}$ & $9^{\circ}$ & 0.809 & $8.74^{\circ}$ & $17.50^{\circ}$ \\
$2^{\circ}$ & 0.990 & $0.42^{\circ}$ & $3.98^{\circ}$ & $10^{\circ}$ & 0.766 & $10.97^{\circ}$ & $19.53^{\circ}$ \\
$3^{\circ}$ & 0.978 & $0.94^{\circ}$ & $5.95^{\circ}$ & $11^{\circ}$ & 0.719 & $13.58^{\circ}$ & $21.68^{\circ}$ \\
$4^{\circ}$ & 0.961 & $1.67^{\circ}$ & $7.90^{\circ}$ & $12^{\circ}$ & 0.669 & $16.64^{\circ}$ & $24.04^{\circ}$ \\
$5^{\circ}$ & 0.940 & $2.62^{\circ}$ & $9.82^{\circ}$ & $13^{\circ}$ & 0.616 & $20.31^{\circ}$ & $26.74^{\circ}$ \\
$6^{\circ}$ & 0.914 & $3.78^{\circ}$ & $11.72^{\circ}$ & $14^{\circ}$ & 0.559 & $24.89^{\circ}$ & $30.03^{\circ}$ \\
$7^{\circ}$ & 0.883 & $5.18^{\circ}$ & $13.63^{\circ}$ & $15^{\circ}$ & 0.500 & $31.22^{\circ}$ & $34.63^{\circ}$ \\
\hline \hline
\end{tabular}

for Bob and Charlie in the case of $P_{A B}>P_{A C}$. The optimal pairs of measurement parameters $\{\alpha, \beta\}$ in different situations are shown in Tables I, II, and III.

\section{APPENDIX B: QUANTIFY THE DEGREE OF MEASUREMENT BIASEDNESS, INCOMPATIBILITY, AND SHARPNESS FROM TWO QRACS}

In our entanglement-assisted sequential QRAC protocol, a maximum two-qubit entanglement state $(|H H\rangle+|V V\rangle) / \sqrt{2}$ is shared among three parties, Alice, Bob, and Charlie, where Alice has access to one qubit, and Bob and Charlie have access to the other qubit. Then, they perform some measurements on her (his) state to encode or decode a message, which in turn can be expressed as

$$
\begin{aligned}
& M_{a \mid \vec{r}_{x}}=\left[I_{A}+(-1)^{a}\left((x \oplus 1) \sigma_{1}+x \sigma_{3}\right)\right] / 2, \\
& M_{b \mid \vec{s}_{y}}=\left[I_{B}+(-1)^{b} \eta_{y}\left(\cos (\alpha) \sigma_{1}+\sin (\alpha) \sigma_{3}\right)\right] / 2, \\
& M_{c \mid \vec{t}_{z}}=\left[I_{C}+(-1)^{c}\left(\cos (\beta) \sigma_{1}+\sin (\beta) \sigma_{3}\right)\right] / 2,
\end{aligned}
$$

where $x=x_{0} \oplus x_{1}, x_{0}\left(x_{1}\right) \in\{0,1\}$ is the bit to be encoded, $\eta_{y} \in[0,1]$ is the sharpness parameter, and $M_{b \mid \vec{s}_{y}}=K_{b \mid \vec{s}_{y}}^{\dagger}$. $K_{b \mid \vec{s}_{y}}, K_{b \mid \vec{s}_{y}}$ is the Kraus operator.

Depending on the choice of measurement and the observed outcome, Alice encodes her two classical bits, $\left\{x_{0}, x_{1}\right\}$, into one of the four possible pure qubit states $\rho_{a \mid \vec{r}_{x}}=\left[I_{a}+\vec{p}_{a \mid \vec{r}_{x}}\right.$. $\vec{\sigma}] / 2$ with equal probability $1 / 4$, whose Bloch vectors can be expressed as

$$
\begin{aligned}
& p_{0 \mid \vec{r}_{0}}=(1,0,0), p_{1 \mid \vec{r}_{0}}=(-1,0,0), \\
& p_{0 \mid \vec{r}_{1}}=(0,0,1), p_{1 \mid \vec{r}_{1}}=(0,0,-1) .
\end{aligned}
$$

After each round of measurement, Alice sends one classical bit $m_{1}=x_{0} \oplus a$ to Bob and Charlie to increase their decoding success probabilities. Because of the symmetry of the

TABLE II. The measurement settings when $\eta_{0}=0.707$.

\begin{tabular}{cccc}
\hline \hline$\theta_{\lambda}$ & $\eta_{1}$ & $\alpha$ & $\beta$ \\
\hline $1^{\circ}$ & 0.998 & $15.52^{\circ}$ & $24.32^{\circ}$ \\
$2^{\circ}$ & 0.990 & $17.15^{\circ}$ & $26.49^{\circ}$ \\
$3^{\circ}$ & 0.978 & $19.26^{\circ}$ & $28.76^{\circ}$ \\
$4^{\circ}$ & 0.961 & $22.00^{\circ}$ & $31.21^{\circ}$ \\
$5^{\circ}$ & 0.940 & $25.61^{\circ}$ & $33.94^{\circ}$ \\
$6^{\circ}$ & 0.914 & $30.80^{\circ}$ & $37.29^{\circ}$ \\
$7^{\circ}$ & 0.883 & $42.60^{\circ}$ & $43.76^{\circ}$ \\
\hline \hline
\end{tabular}

TABLE III. The measurement settings when $\eta_{0}=\eta_{1}=\eta$.

\begin{tabular}{cccr}
\hline \hline$\theta_{\lambda}$ & $\eta_{1}$ & $\alpha$ & $\beta$ \\
\hline $2^{\circ}$ & 0.990 & $1.12^{\circ}$ & $7.94^{\circ}$ \\
$3^{\circ}$ & 0.978 & $2.52^{\circ}$ & $11.85^{\circ}$ \\
$4^{\circ}$ & 0.961 & $4.53^{\circ}$ & $15.72^{\circ}$ \\
$5^{\circ}$ & 0.940 & $7.23^{\circ}$ & $19.60^{\circ}$ \\
$6^{\circ}$ & 0.914 & $10.79^{\circ}$ & $23.59^{\circ}$ \\
$7^{\circ}$ & 0.883 & $15.57^{\circ}$ & $27.83^{\circ}$ \\
$8^{\circ}$ & 0.848 & $22.40^{\circ}$ & $32.70^{\circ}$ \\
\hline \hline
\end{tabular}

initial sharing states, Bob's corresponding condition state will be the same as Alice's encoding state. However, due to the influence of Bob's measurements, the state sharing between Alice and Charlie will be decohered to $\rho_{A C}=\frac{1}{2} \sum_{y, b}\left(I_{A} \otimes\right.$ $\left.K_{b \mid \vec{s}_{y}}\right) \rho_{A B}\left(I_{A} \otimes K_{b \mid \vec{s}_{y}}^{\dagger}\right)$. Thus Charlie's condition state becomes $\rho_{a \mid \vec{r}_{x}}=\frac{1}{2} \sum_{y, b} K_{b \mid \vec{s}_{y}} \rho_{a \mid \vec{r}_{x}} K_{b \mid \vec{s}_{y}}^{\dagger}=\frac{1}{2}\left[I_{c}+\vec{m}_{a \mid \vec{r}_{x}} \cdot \vec{\sigma}\right]$, where $\vec{m}_{a \mid \vec{r}_{x}}$ is the corresponding Bloch vector, which reads as

$$
\begin{aligned}
& \vec{m}_{0 \mid \vec{r}_{0}}=(F \sin (2 \alpha), 0, G \cos (2 \alpha)), \\
& \vec{m}_{1 \mid \vec{r}_{0}}=(-F \sin (2 \alpha), 0,-G \cos (2 \alpha)), \\
& \vec{m}_{0 \mid \vec{r}_{1}}=(G \cos (2 \alpha), 0, F \sin (2 \alpha)), \\
& \vec{m}_{1 \mid \vec{r}_{1}}=(-G \cos (2 \alpha), 0,-F \sin (2 \alpha)),
\end{aligned}
$$

TABLE IV. Details of the results presented in the main text when the measurement directions of Bob and Charlie both are set at $\alpha=\beta=\pi / 4$. The angle of the half-wave plates, $\theta_{\lambda}$, is used to tune the sharpness of Bob's effective measurement whose theoretical value is equal to $\cos \left(4 \theta_{\lambda}\right)$. $\eta_{\text {low }}$ and $\eta_{\text {up }}$ are the corresponding experimental sharpness lower and upper bounds obtained by Eq. (B8) with the success average probability listed on the right side. $P_{M B 0}$ $\left(P_{M C 0}\right)$ represents the experimental average probability when Bob (Charlie) successfully guessed Alice's first bit $x_{0}$ with the corresponding measurement sharpness. Similarly, $P_{M B 1}\left(P_{M C 1}\right)$ represents the experimental average probability when Bob (Charlie) successfully guessed Alice's second bit $x_{1}$. Error bars are estimated by the Poissonian statistics of two-photon coincidences which are about 0.001 for the measurement sharpness and about 0.002 for the average success probability.

\begin{tabular}{lcccccc}
\hline \hline$\theta_{\lambda}$ & $\eta_{\text {low }}$ & $\eta_{\text {up }}$ & $P_{M B 0}$ & $P_{M B 1}$ & $P_{M C 0}$ & $P_{M C 1}$ \\
\hline $0^{\circ}$ & 0.997 & 1.000 & 0.858 & 0.856 & 0.677 & 0.674 \\
$2^{\circ}$ & 0.964 & 0.995 & 0.842 & 0.840 & 0.693 & 0.696 \\
$3^{\circ}$ & 0.953 & 0.976 & 0.841 & 0.833 & 0.730 & 0.700 \\
$4^{\circ}$ & 0.945 & 0.968 & 0.838 & 0.830 & 0.715 & 0.728 \\
$5^{\circ}$ & 0.914 & 0.942 & 0.836 & 0.810 & 0.738 & 0.734 \\
$6^{\circ}$ & 0.905 & 0.913 & 0.820 & 0.820 & 0.735 & 0.762 \\
$7^{\circ}$ & 0.882 & 0.888 & 0.815 & 0.810 & 0.761 & 0.756 \\
$8^{\circ}$ & 0.826 & 0.853 & 0.791 & 0.792 & 0.775 & 0.762 \\
$10^{\circ}$ & 0.757 & 0.756 & 0.773 & 0.762 & 0.781 & 0.796 \\
$12^{\circ}$ & 0.652 & 0.672 & 0.732 & 0.729 & 0.794 & 0.821 \\
$14^{\circ}$ & 0.587 & 0.597 & 0.710 & 0.705 & 0.821 & 0.816 \\
$16^{\circ}$ & 0.411 & 0.452 & 0.648 & 0.643 & 0.840 & 0.839 \\
$18^{\circ}$ & 0.300 & 0.348 & 0.621 & 0.591 & 0.826 & 0.826 \\
$20^{\circ}$ & 0.151 & 0.221 & 0.554 & 0.553 & 0.848 & 0.850 \\
$22.5^{\circ}$ & 0.015 & 0.017 & 0.481 & 0.507 & 0.843 & 0.857 \\
\hline \hline
\end{tabular}


TABLE V. The average success probability when $\eta_{0}=\eta_{1}=\eta$. $P_{A B}$ and $P_{A C}$ are obtained with $\alpha=\beta=\pi / 4 . P_{A B}^{\text {opt }}$ and $P_{A C}^{\text {opt }}$ are obtained when Bob and Charlie further optimize their measurement directions by maximizing the minimum value of $\left\{P_{A B}, P_{A C}\right\}$; the corresponding values of $\alpha$ and $\beta$ are shown in Table III. Error bars are estimated by the Poissonian statistics of two-photon coincidences which are about 0.002 .

\begin{tabular}{cccccc}
\hline \hline$\theta_{\lambda}$ & $\eta$ & $P_{A B}$ & $P_{A C}$ & $P_{A B}^{\mathrm{opt}}$ & $P_{A C}^{\mathrm{opt}}$ \\
\hline $2^{\circ}$ & 0.990 & 0.841 & 0.695 & 0.753 & 0.751 \\
$3^{\circ}$ & 0.978 & 0.837 & 0.715 & 0.753 & 0.750 \\
$4^{\circ}$ & 0.961 & 0.834 & 0.721 & 0.757 & 0.758 \\
$5^{\circ}$ & 0.940 & 0.823 & 0.736 & 0.760 & 0.760 \\
$6^{\circ}$ & 0.914 & 0.820 & 0.749 & 0.762 & 0.761 \\
$7^{\circ}$ & 0.883 & 0.812 & 0.758 & 0.770 & 0.767 \\
$8^{\circ}$ & 0.848 & 0.792 & 0.769 & 0.771 & 0.771 \\
\hline \hline
\end{tabular}

where $F=\frac{1}{4}\left(\sqrt{1-\eta_{2}^{2}}-\sqrt{1-\eta_{1}^{2}}\right), G=\frac{1}{4}\left(2+\sqrt{1-\eta_{1}^{2}}+\right.$ $\sqrt{1-\eta_{2}^{2}}+\left(2-\sqrt{1-\eta_{1}^{2}}-\sqrt{1-\eta_{2}^{2}}\right)$.
TABLE VI. The average success probability when $\eta_{0}=0.707$ and $\eta_{1}=\cos \left(4 \theta_{\lambda}\right) . P_{A B}, P_{A C}$, and $P_{A B C}$ are obtained with $\alpha=\beta=$ $\pi / 4$. $P_{A B}^{\mathrm{opt}}, P_{A C}^{\mathrm{opt}}$, and $P_{A B C}^{\mathrm{opt}}$ are obtained when Bob and Charlie further optimize their measurement directions by maximizing the minimum value of $\left\{P_{A B}, P_{A C}\right\}$; the corresponding values of $\alpha$ and $\beta$ are shown in Table II. Error bars are estimated by the Poissonian statistics of two-photon coincidences which are about 0.002 .

\begin{tabular}{ccccccc}
\hline \hline$\theta_{\lambda}$ & $P_{A B}$ & $P_{A C}$ & $P_{A B C}$ & $P_{A B}^{\mathrm{opt}}$ & $P_{A C}^{\mathrm{opt}}$ & $P_{A B C}^{\mathrm{opt}}$ \\
\hline $0^{\circ}$ & 0.799 & 0.749 & 0.753 & 0.748 & 0.752 & 0.484 \\
$2^{\circ}$ & 0.791 & 0.755 & 0.430 & 0.765 & 0.765 & 0.487 \\
$4^{\circ}$ & 0.786 & 0.761 & 0.437 & 0.771 & 0.773 & 0.490 \\
$6^{\circ}$ & 0.781 & 0.778 & 0.442 & 0.778 & 0.780 & 0.494 \\
\hline \hline
\end{tabular}

Finally, Bob and Charlie perform appropriate measurements respectively on their own states and compare their measurement results with the bit $m_{1}$ received from Alice to determine their success. The expected average success probabilities of Bob and Charlie, $P_{A B}$ and $P_{A C}$, can be expressed as

$$
\begin{aligned}
P_{A B}= & \frac{1}{8} \sum_{x, y} P\left(x_{y}=m_{1} \oplus b \mid x, y\right), \quad=\frac{1}{2}+\frac{1}{16}\left[\left(\vec{p}_{0 \mid \vec{r}_{0}}-\vec{p}_{1 \mid \vec{r}_{0}}\right) \cdot\left(\eta_{0} \vec{s}_{0}+\eta_{1} \vec{s}_{1}\right)\right]+\frac{1}{16}\left[\left(\vec{p}_{0 \mid \vec{r}_{1}}-\vec{p}_{1 \mid \vec{r}_{1}}\right) \cdot\left(\eta_{0} \vec{s}_{0}-\eta_{1} \vec{s}_{1}\right)\right], \\
= & \frac{1}{8}\left[\left(4+\left(\eta_{0}+\eta_{1}\right)\right)(\cos \alpha+\sin \alpha)\right], \quad P_{A C}=\frac{1}{8} \sum_{x, z} P\left(x_{z}=m_{1} \oplus c \mid x, z\right), \quad=\frac{1}{2}+\frac{1}{16}\left[\left(\vec{m}_{0 \mid \vec{r}_{0}}-\vec{m}_{1 \mid \vec{r}_{0}}\right) \cdot\left(\vec{t}_{0}+\vec{t}_{1}\right)\right] \\
& +\frac{1}{16}\left[\left(\vec{m}_{0 \mid \vec{r}_{1}}-\vec{m}_{1 \mid \vec{r}_{1}}\right) \cdot\left(\vec{t}_{0}-\vec{t}_{1}\right)\right], \quad=\frac{1}{8}\left[4+2 M+N\left(\sqrt{1-\eta_{0}^{2}}+\sqrt{1-\eta_{1}^{2}}\right)\right],
\end{aligned}
$$

where $M=\cos ^{2} \alpha \cos \beta+\sin ^{2} \alpha \sin \beta, \quad N=\cos ^{2} \alpha \sin \beta+$ $\sin ^{2} \alpha \cos \beta, \alpha, \beta \in[0, \pi / 4]$. We defined the biasedness of each decoder's measurement settings by their overlap $\vec{s}_{0}$. $\vec{s}_{1}$ and $\vec{t}_{0} \cdot \vec{t}_{1}$. Theoretically, $\left|\vec{s}_{0} \cdot \vec{s}_{1}\right|=\cos (2 \alpha)$ and $\left|\vec{t}_{0} \cdot \vec{t}_{1}\right|=$ $\cos (2 \beta)$ for Bob and Charlie, where $\alpha=\pi / 4(\beta=\pi / 4)$ corresponds to mutually unbiased measurement.

It is obvious that the success probability depends on the degree of measurement sharpness and biasedness. Therefore, by properly adjusting the measurement biasedness parameters, the useful range of sharpness parameters to make both QRACs outperform their classical counterparts can be expanded. Now we would like to establish the relationship between the degree of measurement biasedness and the average success probability for both Bob and Charlie; thus we can quantify them from these two QRACs. According to Ref. [13], for a pair of our fixed qubit observables, the maximum quantum value of the QRAC for Alice and Bob satisfies

$$
P_{A B} \leqslant \frac{1}{2}+\frac{1}{16}(\sqrt{2 \mu+2 v}+\sqrt{2 \mu-2 v}),
$$

where $\mu=2\left(\eta_{0}^{2}+\eta_{1}^{2}\right)$ and $v=4 \eta_{0} \eta_{1} \vec{s}_{0} \cdot \vec{s}_{1}$. We can easily obtain that

$$
\left|\vec{s}_{0} \cdot \vec{s}_{1}\right| \leqslant \frac{8 P_{A B}-4}{\eta_{1}+\eta_{2}} \sqrt{2-\left(\frac{8 P_{A B}-4}{\eta_{1}+\eta_{2}}\right)^{2}} .
$$

Combining Eq. (3) and Eq. (B6), we can also quantify the bound of $\left|\vec{t}_{0} \cdot \vec{t}_{1}\right|$, whose value depends on $\eta_{0}, \eta_{1}, P_{A B}, P_{A C}$, and $\left|\vec{s}_{0} \cdot \vec{s}_{1}\right|$.

On the other hand, the degree of measurement incompatibility for Bob and Charlie can be expressed as $D\left(\vec{s}_{0} \cdot \vec{s}_{1}\right)=\left|\eta_{0} \vec{s}_{0}+\eta_{1} \vec{s}_{1}\right|+\left|\eta_{0} \vec{s}_{0}-\eta_{1} \vec{s}_{1}\right|$ and $D\left(\vec{t}_{0} \cdot \vec{t}_{1}\right)=$ $\left|\vec{t}_{0}+\vec{t}_{1}\right|+\left|\vec{t}_{0}-\vec{t}_{1}\right|$, respectively, which satisfy the following conditions [15]:

$$
D\left(\vec{s}_{0} \cdot \vec{s}_{1}\right) \geqslant 8 P_{A B}-6, \quad D\left(\vec{t}_{0} \cdot \vec{t}_{1}\right) \geqslant \frac{16 P_{A C}-8}{m}-2,
$$

where $m$ represents the maximum value of $\left\{\left|\vec{m}_{0 \mid \vec{r}_{0}}-\vec{m}_{1 \mid \vec{r}_{0}}\right|\right.$, $\mid \vec{m}_{0 \mid \vec{r}_{1}}-\vec{m}_{\left.1\left|\vec{r}_{1}\right|\right\}}$

TABLE VII. The experimental data for Fig. 3(e) in the main text. Error bars are estimated by the Poissonian statistics of two-photon coincidences which are about 0.003 .

\begin{tabular}{cccccc}
\hline \hline$\theta_{\lambda}$ & $\eta$ & $\left|\vec{s}_{0} \cdot \vec{s}_{1}\right|$ & $D\left(\vec{s}_{0} \cdot \vec{s}_{1}\right)$ & $\left|\vec{t}_{0} \cdot \vec{t}_{1}\right|$ & $D\left(\vec{t}_{0} \cdot \vec{t}_{1}\right)$ \\
\hline $2^{\circ}$ & 0.990 & 0.999 & 0.019 & 0.962 & 0.030 \\
$3^{\circ}$ & 0.978 & 0.996 & 0.040 & 0.916 & 0.036 \\
$4^{\circ}$ & 0.961 & 0.987 & 0.068 & 0.853 & 0.072 \\
$5^{\circ}$ & 0.940 & 0.968 & 0.101 & 0.775 & 0.106 \\
$6^{\circ}$ & 0.914 & 0.930 & 0.137 & 0.680 & 0.121 \\
$7^{\circ}$ & 0.883 & 0.856 & 0.175 & 0.564 & 0.218 \\
$8^{\circ}$ & 0.848 & 0.710 & 0.214 & 0.416 & 0.292 \\
\hline \hline
\end{tabular}


TABLE VIII. The experimental data for Fig. 4(a) in the main text. Error bars are estimated by the Poissonian statistics of twophoton coincidences which are about 0.002 .

\begin{tabular}{lcccc}
\hline \hline$\theta_{\lambda}$ & $\eta$ & $I_{A B}$ & $I_{A C}$ & $H_{\min }$ \\
\hline $0^{\circ}$ & 1.000 & 2.855 & 1.405 & 0.974 \\
$2^{\circ}$ & 0.990 & 2.727 & 1.560 & 0.540 \\
$4^{\circ}$ & 0.961 & 2.675 & 1.770 & 0.454 \\
$6^{\circ}$ & 0.913 & 2.560 & 1.985 & 0.320 \\
$8^{\circ}$ & 0.848 & 2.332 & 2.149 & 0.211 \\
$10^{\circ}$ & 0.766 & 2.141 & 2.308 & 0.194 \\
$12^{\circ}$ & 0.669 & 1.844 & 2.461 & 0.237 \\
$14^{\circ}$ & 0.559 & 1.660 & 2.548 & 0.310 \\
$16^{\circ}$ & 0.438 & 1.164 & 2.645 & 0.414 \\
$18^{\circ}$ & 0.309 & 0.851 & 2.730 & 0.545 \\
$20^{\circ}$ & 0.173 & 0.427 & 2.794 & 0.711 \\
$22.5^{\circ}$ & 0.001 & 0.047 & 2.830 & 0.999 \\
\hline \hline
\end{tabular}

In order to get a better upper bound or lower bound for the above parameters, we should take all kinds of experimental imperfection into consideration. That is to say, all the parameters in Eq. (B6), and Eq. (B7) should use experimental data. Since the exact measurement sharpness parameter is unknown to us, we also need to estimate its value with the observed success probability. It has been demonstrated that its bounds can be determined as follows [28]:

$$
\begin{aligned}
& \eta \geqslant \eta_{\text {low }}=\sqrt{2}\left(2 P_{A B}-1\right), \\
& \eta \leqslant \eta_{\text {up }}=2 \sqrt{\left(2+\sqrt{2}-4 P_{A C}\right)\left(2 P_{A C}-1\right) .}
\end{aligned}
$$

Then, substituting its minimum value into Eq. (B6) and the maximum value into Eq. (B7), respectively, we can obtain the corresponding upper bound and lower bound for the degree of measurement biasedness and incompatibility.

\section{APPENDIX C: ERROR ESTIMATION}

In our experiment, the statistics of photon counts are assumed to follow a Poisson distribution. We use a subprogram of Poisson distribution in Wolfram MATHEMATICA 11.2 to simulate the Poisson distribution. The values of each measurement quantity are then calculated from 50 randomly grouped counting sets, in which the error of the quantity is estimated by the standard deviation (SD). To obtain the error of the final value we want (e.g., $P_{A B}, P_{A C}$, etc.), we sum all the errors of the measured quantities included in the equation to calculate the final value. For example, the error of $P_{A B}$ and $P_{A C}$ are calculated as

$$
\begin{aligned}
& \mathrm{SD}\left(P_{A B}\right)=\frac{1}{8} \sum_{x, y} \operatorname{SD}\left(P\left(x_{y}=m_{1} \oplus b \mid x, y\right)\right), \\
& \mathrm{SD}\left(P_{A C}\right)=\frac{1}{8} \sum_{x, z} \operatorname{SD}\left(P\left(x_{z}=m_{1} \oplus c \mid x, z\right)\right) .
\end{aligned}
$$

\section{APPENDIX D: EXPERIMENTAL DATA}

Tables IV-VIII give details of the experimental data and results from the main text.
[1] A. Ambainis, A. Nayak, A. Ta-Shma, and U. Vazirani, Dense quantum coding and a lower bound for 1-way quantum automata, in Proceedings of the Thirty-First Annual ACM Symposium on Theory of Computing (ACM, New York, 1999), pp. 376-383.

[2] R. W. Spekkens, D. H. Buzacott, A. J. Keehn, B. Toner, and G. J. Pryde, Preparation Contextuality Powers Parity-Oblivious Multiplexing, Phys. Rev. Lett. 102, 010401 (2009).

[3] M. Hayashi, K. Iwama, H. Nishimura, R. Raymond, and S. Yamashita, (4, 1)-Quantum random access coding does not exist-one qubit is not enough to recover one of four bits, New J. Phys. 8, 129 (2006).

[4] A. Ambainis, D. Leung, L. Mancinska, and M. Ozols, Quantum random access codes with shared randomness, arXiv:0810.2937.

[5] M. Pawlowski and M. Źukowski, Entanglement-assisted random access codes, Phys. Rev. A 81, 042326 (2010).

[6] X. R. Wang, L. Y. Wu, C. X. Liu, T. J. Liu, J. Li, and Q. Wang, Experimental generation of entanglement-assisted quantum random access code, Phys. Rev. A 99, 052313 (2019).

[7] A. Tavakoli, A. Hameedi, B. Marques, and M. Bourennane, Quantum Random Access Codes Using Single D-Level Systems, Phys. Rev. Lett. 114, 170502 (2015).

[8] M. Hayashi, K. Iwama, H. Nishimura, R. Raymond, and S. Yamashita, Quantum Network Coding (Springer, Berlin, 2007), pp. 610-621.
[9] M. Pawlowski and N. Brunner, Semi-device-independent security of one-way quantum key distribution, Phys. Rev. A 84, 010302(R) (2011).

[10] A. Chaturvedi, M. Ray, R. Veynar, and M. Pawlowski, On the security of semi-device-independent QKD protocols, Quantum Inf. Process. 17, 131 (2018).

[11] H. W. Li, M. Pawlowski, Z. Q. Yin, G. C. Guo, and Z. F. Han, Semi-device-independent randomness certification using $n \rightarrow 1$ quantum random access codes, Phys. Rev. A 85, 052308 (2012).

[12] E. A. Aguilar, M. Farkas, D. Martínez, M. Alvarado, J. Cariñe, G. B. Xavier, J. F. Barra, G. Cañas, M. Pawlowski, and G. Lima, Certifying an Irreducible 1024-Dimensional Photonic State Using Refined Dimension Witnesses, Phys. Rev. Lett. 120, 230503 (2018).

[13] A. Tavakoli, J. Kaniewski, T. Vértesi, D. Rosset, and N. Brunner, Self-testing quantum states and measurements in the prepare-and-measure scenario, Phys. Rev. A 98, 062307 (2018).

[14] M. Farkas and J. Kaniewski, Self-testing mutually unbiased bases in the prepare-and-measure scenario, Phys. Rev. A 99, 032316 (2019).

[15] H. Anwer, S. Muhammad, W. Cherifi, N. Miklin, A. Tavakoli, and M. Bourennane, Experimental Characterization of Unsharp Qubit Observables and Sequential Measurement Incompatibility via Quantum Random Access Codes, Phys. Rev. Lett. 125, 080403 (2020). 
[16] R. Silva, N. Gisin, Y. Guryanova, and S. Popescu, Multiple Observers can Share the Nonlocality of Half of an Entangled Pair by Using Optimal Weak Measurements, Phys. Rev. Lett. 114, 250401 (2015)

[17] M. J. Hu, Z. Y. Zhou, X. M. Hu, C. F. Li, G. C. Guo, and Y. S. Zhang, Experimental sharing of nonlocality among multiple observers with one entangled pair via optimal weak measurements, npj Quantum Inf. 4, 63 (2018).

[18] G. Foletto, L. Calderaro, A. Tavakoli, M. Schiavon, F. Picciariello, A. Cabello, P. Villoresi, and G. Vallone, Experimental Demonstration of Sustained Entanglement and Nonlocality after Sequential Measurements, Phys. Rev. Appl. 13, 044008 (2020).

[19] P. J. Brown and R. Colbeck, Arbitrarily Many Independent Observers Can Share the Nonlocality of a Single Maximally Entangled Qubit Pair, Phys. Rev. Lett. 125, 090401 (2020).

[20] C. L. Ren, T. F. Feng, D. Yao, J. L. Chen, and X. Q. Zhou, Non-locality can be shared between Alice and three Bobs in unbiased settings case, arXiv:2006.16462.

[21] Y. Kim, Y. S. Kim, S. Y. Lee, S. W. Han, S. Moon, Y. H. $\mathrm{Ki}$, and Y. W. Cho, Direct quantum process tomography via measuring sequential weak values of incompatible observables, Nat. Commun. 9, 192 (2018).

[22] F. Piacentini, A. Avella, M. P. Levi, M. Gramegna, G. Brida, I. P. Degiovanni, E. Cohen, R. Lussana, F. Villa, A. Tosi, F. Zappa, and M. Genovese, Measuring Incompatible Observables by Exploiting Sequential Weak Values, Phys. Rev. Lett. 117, 170402 (2016).

[23] M. Pusey, Anomalous Weak Values are Proofs of Contextuality, Phys. Rev. Lett. 113, 200401 (2014).

[24] F. Piacentini, A. Avella, M. P. Levi, R. Lussana, F. Villa, A. Tosi, F. Zappa, M. Gramegna, G. Brida, I. P. Degiovanni, and M. Genovese, Experiment Investigating the Connection between Weak Values and Contextuality, Phys. Rev. Lett. 116, 180401 (2016).

[25] A. Bera, S. Mal, A. Sen(De), and U. Sen, Witnessing bipartite entanglement sequentially by multiple observers, Phys. Rev. A 98, 062304 (2018).
[26] Y. Choi, S. Hong, T. Pramanik, H. Lim, Y. Kim, H. Jung, S. Han, S. Moon, and Y. Cho, Demonstration of simultaneous quantum steering by multiple observers via sequential weak measurements, Optica 7, 675 (2020).

[27] S. Datta and A. S. Majumdar, Sharing of nonlocal advantage of quantum coherence by sequential observers, Phys. Rev. A 98, 042311 (2018).

[28] K. Mohan, A. Tavakoli, and N. Brunner, Sequential random access codes and self-testing of quantum measurement instruments, New J. Phys. 21, 083034 (2019).

[29] G. Foletto, L. Calderaro, G. Vallone, and P. Villoresi, Experimental demonstration of sequential quantum random access codes, Phys. Rev. Research 2, 033205 (2020).

[30] A. Hameedi, D. Saha, P. Mironowicz, M. Pawlowski, and M. Bourennane, Complementarity between entanglement-assisted and quantum distributed random access code, Phys. Rev. A 95, 052345 (2017).

[31] S. Pironio, A. Acin, S. Massar, A. B. Giroday, D. N. Matsukevich, P. Maunz, S. Olmschenk, D. Hayes, L. Luo, T. A. Manning, and C. Monroe, Random numbers certified by Bell's theorem, Nature (London) 464, 1021 (2010).

[32] A. Fedrizzi, T. Herbst, A. Poppe, T. Jennewein, and A. Zeilinger, A wavelength-tunable fiber-coupled source of narrowband entangled photons, Opt. Express 15, 15377 (2007).

[33] X.-B. An, H.-W. Li, Z.-Q. Yin, M.-J. Hu, W. Huang, B.- J. Xu, S. Wang, W. Chen, G.-C. Guo, and Z.-F. Han, Experimental three-party quantum random number generator based on dimension witness violation and weak measurement, Opt. Lett. 43 , 3437 (2018).

[34] A. Tavakoli and R. Uola, Measurement incompatibility and steering are necessary and sufficient for operational contextuality, Phys. Rev. Research 2, 013011 (2020).

[35] C. Carmeli, T. Heinosaari, and A. Toigo, Quantum random access codes and incompatibility of measurements, Europhys. Lett. 130, 50001 (2020).

[36] S. Designolle, P. Skrzypczyk, F. Fröwis, and N. Brunner, Quantifying Measurement Incompatibility of Mutually Unbiased Bases, Phys. Rev. Lett. 122, 050402 (2019). 